\title{
Estado Nutricional e Fatores Associados em Adolescentes de Campo Grande (MS)
} Nutritional status and associated factors in adolescents of Campo Grande (MS)

\author{
CRISTINA CORREADA SILVALUBAS ${ }^{1}$ \\ MARJORIE CRISTINA CALIANI DE QUEIROZ' \\ GIOVANAELIZAPEGOLO²
}

\section{RESUMO}

Objetivos: Avaliar o estado nutricional e fatores associados em adolescentes. Materiais e métodos: Estudo transversal com adolescentes (14 anos) de uma escola pública de Campo Grande (MS). As variáveis investigadas foram: classificação econômica; estado nutricional; atividade física; ingestão de energia, macro e micronutrientes e hábitos alimentares. Resultados: Participaram do estudo 50 adolescentes (62\%, sexo feminino). Destes, $26 \%$ apresentaram excesso de peso ( $26,3 \% \mathrm{H}$ meninos; $25,8 \% \mathrm{H}$ meninas). A prática de atividade física foi mencionada por $100 \%$ dos meninos e por $77,4 \%$ das meninas. Não houve diferença significativa $(p<0,05)$ entre estado nutricional e prática de atividade física. Em relação ao consumo de energia, 34\% apresentaram consumo acima da Necessidade Estimada de Energia. Elevado percentual de adolescentes apresentaram reduzida ingestão de lipídeos $(61,7 \%)$, cálcio $(55,3 \%)$, fibras $(80,9 \%)$ e vitamina A $(93,6 \%)$ e $34 \%$ dos adolescentes consumiram sódio acima do limite tolerável. Reduzida proporção de adolescentes relataram consumo diário de frutas $(12 \%)$ e salada crua $(18 \%)$. Porém, $30 \%$ relataram consumo diário de doces e $36 \%$ consumo de refrigerantes de 3 a 4 vezes/semana. Conclusões: A proporção de adolescentes com excesso de peso mostrou-se expressiva. A atividade física insuficiente, aliada a ingestão inadequada de energia e nutrientes, pautada por hábitos alimentares prejudiciais, evidenciam fatores de risco para Doenças Crônicas Não Transmissíveis com instalação precoce.

\section{DESCRITORES}

Adolescente. Atividade física. Cálcio. Consumo de alimentos. Estado nutricional.

\begin{abstract}
Objective: To assess the nutritional status and associated factors in adolescents. Material and Methods: This was a cross-sectional study with adolescents (14 years old) of a public school of Campo Grande (MS). The variables investigated were: socio-economic status; nutritional status; physical activity; energy, macro and micronutrients intake and eating habits. Results: The participants were 50 adolescents $(62 \%$, female gender). Of these, $26 \%$ were overweight $(26.3 \%$ " boys; $25.8 \%$ " girls). The practice of physical activity was reported by $100 \%$ of the boys and $77.4 \%$ of the girls. There was no significant difference $(p<0.05)$ between nutritional status and practice of physical activity. Regarding energy intake, $34 \%$ of the participants were above the Estimated Energy Requirements by age and weight. A high percentage of adolescents showed reduced intake of lipids $(61.7 \%)$, calcium $(55.3 \%)$, fibers $(80.9 \%)$ and vitamin $\mathrm{A}(93.6 \%)$, and $34 \%$ of the adolescents had an intake level of sodium above the recommended dietary intake. A small percentage of adolescents reported daily intake of fruits $(12 \%)$ and raw salad $(18 \%)$. However, $30 \%$ reported daily intake of sweets and $36 \%$ of soft drinks 3 or 4 times/week. Conclusions: The proportion of overweight adolescents was found to be expressive. Lack of physical activity associated with inadequate intake of energy and nutrients in a context of bad eating habits indicate risk factors for Chronic NonCommunicable Diseases with early onset.
\end{abstract}

\section{DESCRIPTORS}

Adolescent. Physical activity. Calcium. Food intake. Nutritional status.

1 Graduanda em Nutrição, Universidade Federal de Mato Grosso do Sul (UFMS), Campo Grande, Mato Grosso do Sul/Brasil.

2 Docente Doutora do Curso de Nutrição, Universidade Federal de Mato Grosso do Sul (UFMS), Campo Grande, Mato Grosso do Sul/Brasil. 
$\mathrm{A}$ adolescência é notadamente importante para a aquisição de práticas alimentares saudáveis, que por sua vez influenciam decisivamente o crescimento/desenvolvimento e o desempenho físico e intelectual. Além disso, podem contribuir para a diminuição do risco de desenvolvimento de Doenças Crônicas Não Transmissíveis (DCNT) e impactar positivamente na qualidade de vida desse grupo populacional ${ }^{1}$. Desta forma, a ingestão adequada de energia e de nutrientes apresenta papel essencial neste processo. Sabe-se que vitaminas e minerais atuam de forma específica e sinergicamente para o suporte das funções metabólicas, porém, a prevalência de deficiências nutricionais entre adolescentes continua a ser uma preocupação entre as ações de nutrição em saúde pública ${ }^{2}$.

A substituição das principais refeições por lanches excessivamente calóricos, bem como a omissão de refeições, como o café da manhã, contribuem para a inadequação do consumo de macro e micronutrientes. Ainda, o consumo rotineiro de alimentos ricos em açúcar, carboidratos refinados e gorduras saturadas, somado ao reduzido consumo de frutas e hortaliças, também tendem a comprometer o perfil nutricional da alimentação $^{3}$.

Além da alimentação, a atividade física representa papel importante para a saúde. Com o processo de urbanização, a frequência e duração de atividades físicas e de lazer diminuíram consideravelmente com o passar dos anos ${ }^{4}$. Soma-se ainda o fato de que adolescentes pouco ativos desenvolvem outros comportamentos não saudáveis, como baixo consumo de verduras e legumes, o que aumenta o risco para o desenvolvimento de $\mathrm{DCNT}^{5}$. Para todos os estágios de vida, níveis adequados de atividade física e dieta saudável auxiliam no controle de peso e, consequentemente, para a prevenção de tais doenças ${ }^{6}$.

Diante do exposto, o objetivo deste estudo foi avaliar o estado nutricional e fatores associados em adolescentes.

\section{MATERIAIS E MÉTODOS}

Trata-se de estudo transversal, com adolescentes matriculados em uma escola pública de Campo Grande (MS). Foram incluídos na amostra adolescentes, de ambos os sexos, com idade a partir de 14 anos completos até 14 anos, 11 meses e 29 dias, que aceitaram participar e apresentaram concordância dos pais ou responsáveis. A obtenção dos dados aconteceu no período de março a junho de 2015.

A classificação econômica foi obtida mediante aplicação do Critério de Classificação Econômica Brasil, desenvolvido pela Associação Brasileira de Empresas de Pesquisa ${ }^{7}$. Este questionário foi respondido pelos pais ou responsável pelo adolescente e foram mantidos os pontos de corte originais $(\mathrm{A} 1, \mathrm{~A} 2=\mathrm{R} \$ 11.037$; $\mathrm{B} 1=$ $\mathrm{R} \$$ 6.006; $\mathrm{B} 2=\mathrm{R} \$ 3.118 ; \mathrm{C} 1=\mathrm{R} \$ 1.865 ; \mathrm{C} 2=\mathrm{R} \$ 1.277 ; \mathrm{D}$, $\mathrm{E}=\mathrm{R} \$$ 895).

A avaliação do estado nutricional foi realizada por meio da aferição de peso e estatura e posterior análise do Índice de Massa Corporal (IMC). As medidas de peso e estatura foram realizadas em duplicata, obtidas a partir da utilização de uma balança digital (marca Tanita ${ }^{\circledR}$, com capacidade para $150 \mathrm{~kg}$ e escala de 100 gramas) e estadiômetro portátil (marca Sanny®, com capacidade de $210 \mathrm{~cm}$ ). O valor médio obtido para cada participante foi usado para o cálculo do IMC. Adotouse como referência a distribuição de IMC para idade, em percentis, preconizado pela Organização Mundial da Saúde $^{8}$, para a faixa de idade de 5 a 19 anos. Adotou-se a expressão excesso de peso para adolescentes com sobrepeso e obesidade (Percentil > 85).

Para mensurar a atividade física foi adotado questionário validado para adolescentes de 14 a 19 anos $^{9}$. Foram considerados suficientemente ativos os adolescentes com prática de atividade física igual ou superior a 300 minutos/semana. Adolescentes com tempo inferior foram considerados insuficientemente $\operatorname{ativos}^{10}$.

Para a avaliação da ingestão de energia e nutrientes foi adotado o Recordatório 24 horas, em dois momentos, em dias não consecutivos, exceto segundafeira, com intervalo de tempo não inferior a um mês ${ }^{11}$. Os Recordatórios foram autopreenchidos com orientação prévia a respeito sobre quantidade e modo de preparo. As pesquisadoras acompanharam toda a etapa de preenchimento e ficaram à disposição para esclarecimentos. O conteúdo de energia e nutrientes foi calculado por meio da utilização do software DietPro $5 i$. Foram analisados os seguintes nutrientes: energia, distribuição de carboidratos, proteínas e lipídeos, fibra total, cálcio, ferro, sódio, vitamina A e vitamina C. O consumo de energia foi classificado em abaixo ou acima do recomendado, considerando-se adequado valores entre 80 e $120 \%$ da Necessidade Estimada de Energia. Os macronutrientes foram analisados mediante comparação com os Intervalos de Distribuição Aceitáveis. Fibras, minerais e vitaminas foram analisados de acordo com os valores recomendados segundo sexo e idade. Para todos os nutrientes estudados foram adotadas como referência as Dietary Reference Intakes (DRIs), do Institute of Medicine $e^{12-17}$.

A investigação dos hábitos alimentares foi realizada a partir de questões adaptadas da pesquisa 
Vigilância de Fatores de Risco e Proteção para Doenças Crônicas por Inquérito Telefônico ${ }^{18}$, implementada pelo Ministério da Saúde.

Para a caracterização da amostra foi realizada análise descritiva das variáveis investigadas. O teste de Shapiro-Wilk foi utilizado para testar a normalidade. As variáveis antropométricas foram submetidas ao Teste $t$ de Student e atividade física ao Teste de MannWhitney. Para comparar os valores médios de energia e dos demais nutrientes por sexo utilizou-se o Teste de Mann-Whitney. Para comparar a adequação de ingestão entre os sexos utilizou-se o Teste do Qui-quadrado, com Correção de Yates quando necessário. O nível de significância adotado foi de $5 \%$.

Este estudo foi aprovado pelo Comitê de Ética em Pesquisa da Universidade Federal de Mato Grosso do Sul (Parecer 912.043). Os participantes e seus pais/ responsáveis foram informados sobre as razões do estudo e a colaboração que se esperava dos mesmos, cuja participação foi validada após assinaturas dos Termos de Assentimento e de Consentimento Livre e Esclarecido.

\section{RESULTADOS}

Participaram do estudo 50 adolescentes de um total de 71 elegíveis, sendo 19 do sexo masculino (38\%) e 31 do sexo feminino (62\%). Dezessete adolescentes desistiram ao longo do estudo e quatro foram excluídos por apresentarem questionários incompletos.

Ao analisar a classificação econômica, $64 \%$ dos adolescentes pertenciam a famílias classificadas na categoria B (B1, 18\%; B2, 46\%), seguindo-se pela classificação C1 (22\%) e C2 (6\%), conforme classificação proposta pelo critério adotado. Quanto à escolaridade, $34 \%$ dos chefes de família possuíam Ensino Médio Completo ou Superior Incompleto e, 28\%, Ensino Superior Completo. Não foi constatada diferença significativa $(p>0,05)$ entre classificação econômica e estado nutricional e entre escolaridade do chefe da família e estado nutricional, considerando os sexos separadamente.

A Tabela 1 apresenta os valores médios em relação à idade, dados antropométricos e de atividade física de acordo com o sexo. Nota-se que a estatura foi maior para os meninos $(p<0,05)$.

Quanto ao estado nutricional, embora $72 \%$ da amostra apresentasse eutrofia, destaca-se o percentual de adolescentes com excesso de peso ( $26 \%$ ), no entanto, não foi constatada diferença significativa entre os sexos (Tabela 2).

A prática de atividade física foi mencionada por $100 \%$ dos meninos e por $77,4 \%$ das meninas, sendo que $63,2 \%$ dos meninos foram classificados como ativos (e"300 minutos semanais) e 38,7\% das meninas foram classificadas nesta categoria. Os meninos mostraramse significativamente mais adeptos de atividades físicas do que as meninas $(p<0,05)$. Porém, não foi constatada diferença significativa entre estado nutricional e prática de atividade física $(p>0,05)$.

Os resultados apresentados na Tabela 3 indicam a distribuição dos adolescentes de acordo com o consumo de energia, nutrientes e sexo. Destaca-se o percentual de adolescentes (34\%) com consumo de energia superior ao recomendado. Entre os meninos, $61,1 \%$ e $88,9 \%$ apresentaram consumo de carboidratos e proteínas adequado. Para as meninas, 58,6\% e 75,9\% mostraram consumo adequado para os nutrientes mencionados. Contudo, $50 \%$ dos meninos e $69 \%$ das meninas mostraram consumo de lipídeos inferior a $25 \%$.

Embora não tenha sido constatada diferença significativa de consumo entre os sexos para as vitaminas e minerais investigados, exceto para o ferro, a distribuição dos adolescentes de acordo com o consumo ressalta proporções elevadas de consumo insuficiente principalmente para o cálcio e vitamina A. No caso do ferro os meninos apresentaram maior consumo, acima da Necessidade Média Estimada, do que as meninas $(p=0,0048)$. Para fibras, $88,9 \%$ dos meninos e $75,9 \%$ das meninas apresentaram consumo insuficiente. Para o sódio, $61,1 \%$ dos meninos e 55,2\% das meninas apresentaram consumo que ultrapassou a ingestão adequada.

A ingestão de nutrientes de acordo com o estado nutricional e sexo (Tabela 4) apontou maior ingestão de carboidratos por meninas com excesso de peso $(p=0,0419)$ em comparação as eutróficas. No entanto, as eutróficas consomem mais lipídeos que meninas com excesso de peso $(p=0,0473)$. Verificou-se diferença significativa no consumo de fibras entre os meninos. Os meninos eutróficos consumiram maior quantidade de fibras que os com excesso de peso $(p=0,0433)$.

Na Tabela 5 são apresentados os percentuais em relação à frequência semanal de consumo dos alimentos selecionados como marcadores de consumo alimentar associados ao seu impacto, favorável ou não, para a saúde. Destaca-se a proporção reduzida de adolescentes que manifestaram consumo diário de frutas $(12 \%)$, de salada crua (18\%) e de leite $(26 \%)$. Por outro lado, o consumo de refrigerantes com frequência de 3 a $4 \mathrm{dias} /$ semana foi relatado por $36 \%$ dos adolescentes e $28 \%$ relataram consumo de 5 a 6 dias/semana. O consumo diário de doces foi mencionado por $30 \%$ da amostra estudada. 


\begin{tabular}{|c|c|c|c|}
\hline Variáveis & $\begin{array}{c}\text { Meninas } \\
(n=31) \\
\text { Média } \pm \text { DP }\end{array}$ & $\begin{array}{c}\text { Meninos } \\
(n=19) \\
\text { Média } \pm \text { DP }\end{array}$ & $p$ \\
\hline Idade (anos) & $14,6 \pm 0,3$ & $14,5 \pm 0,2$ & 0,3388 \\
\hline Peso (kq) & $56,4 \pm 14,7$ & $60,9 \pm 13,0$ & 0,2804 \\
\hline Estatura $(\mathrm{cm})$ & $1,60 \pm 0,06$ & $1,67 \pm 0,05$ & $0,0002^{*}$ \\
\hline Índice de Massa Corporal $\left(\mathrm{Kg} / \mathrm{m}^{2}\right)$ & $21,9 \pm 5,0$ & $21,7 \pm 3,8$ & 0,8978 \\
\hline Atividade física (minutos) ${ }^{* *}$ & $337,4 \pm 412,4$ & $531,8 \pm 698,6$ & 0.1033 \\
\hline
\end{tabular}

\begin{tabular}{|c|c|c|c|c|c|c|}
\hline \multirow{3}{*}{ Estado nutricional } & \multicolumn{4}{|c|}{ Sexo } & \multirow{2}{*}{\multicolumn{2}{|c|}{ Total }} \\
\hline & \multicolumn{2}{|c|}{$\begin{array}{c}\text { Meninos } \\
(n=19)\end{array}$} & \multicolumn{2}{|c|}{$\begin{array}{c}\text { Meninas } \\
(n=31)\end{array}$} & & \\
\hline & $\mathbf{n}$ & $\%$ & $\mathbf{n}$ & $\%$ & $\mathrm{n}$ & $\%$ \\
\hline Baixo peso & 0 & 0,0 & 1 & 3,2 & 1 & 2,0 \\
\hline Eutrofia & 14 & 73,7 & 22 & 71,0 & 36 & 72,0 \\
\hline Excesso de peso & 05 & 26,3 & 8 & 25,8 & 13 & 26,0 \\
\hline
\end{tabular}

\section{DISCUSSÃO}

Nesta pesquisa constatou-se expressiva proporção $(26 \%)$ de adolescentes com excesso de peso, sendo $26,3 \%$ entre os meninos e $25,8 \%$ entre as meninas. De acordo com a Pesquisa de Orçamentos Familiares (POF 2008-2009) ${ }^{19}$, considerando os anos decorridos desde a Pesquisa Nacional de Despesa Familiar de 19741975, o aumento do percentual de adolescentes com excesso de peso foi constante nos últimos 34 anos. Os resultados da POF 2008-2009 mostraram que entre adolescentes com idades entre 14 e 15 anos, 21,6\% apresentavam excesso de peso. Em um estudo mais recente, com adolescentes de Minas Gerais, com idades entre 6 e 17 anos, constatou-se que 15,4\% estavam acima do peso, destes, $16 \%$ eram obesos e $4,6 \%$ severamente obesos ${ }^{20}$.

A preocupação em relação ao acúmulo de gordura corporal em jovens fundamenta-se especialmente por representar importante fator de risco para doenças DCNT. Complicações do excesso de peso incluem diabetes, doenças cardiovasculares e câncer ${ }^{21}$. Além disso, a obesidade na adolescência aumenta em $80 \%$ a chance de na vida adulta esta condição persistir ${ }^{4}$.

Neste contexto, a prática de atividade física representa uma estratégia com diversos efeitos positivos para a saúde durante a adolescência. A prática regular promove redução do risco para diabetes, dislipidemias/doenças cardiovasculares, depressão e contribui para o controle do peso corporal ${ }^{6}$. No presente estudo, embora a maioria dos adolescentes tenha declarado praticar alguma atividade, tal constatação não indica adequação em relação ao tempo, pois $36,8 \%$ dos meninos e $61,3 \%$ das meninas foram classificados como insuficientemente ativos (d"300 minutos/semana). A proporção de adolescentes insuficientemente ativos encontrada na presente pesquisa aproxima-se da proporção encontrada entre adolescentes de João Pessoa (PB) ${ }^{9}$. Segundo o referido estudo, $31,3 \%$ dos meninos e $60,5 \%$ das meninas foram classificados como insuficientemente ativos, considerando a mesma faixa de idade.

As principais constatações quanto à ingestão de energia e nutrientes apontam situação de risco nutricional para o grupo estudado. De forma geral, cerca de um terço dos adolescentes apresentaram consumo de energia superior ao preconizado. Para os lipídeos, independente do estado nutricional, substancial número de adolescentes (50\% dos meninos e $69 \%$ das meninas) ingeriram valores inferiores à recomendação mínima de $25 \%$ do Valor Energético Total e superior ao recomendado para carboidratos $(29,8 \%)$. Tal fato pode ser justificado pela elevada ingestão de açúcares e carboidratos refinados, decorrente de produtos industrializados.

Estudo realizado com adolescentes de escolas públicas de Minas Gerais, com o objetivo de identificar o consumo alimentar, também encontrou alto consumo 


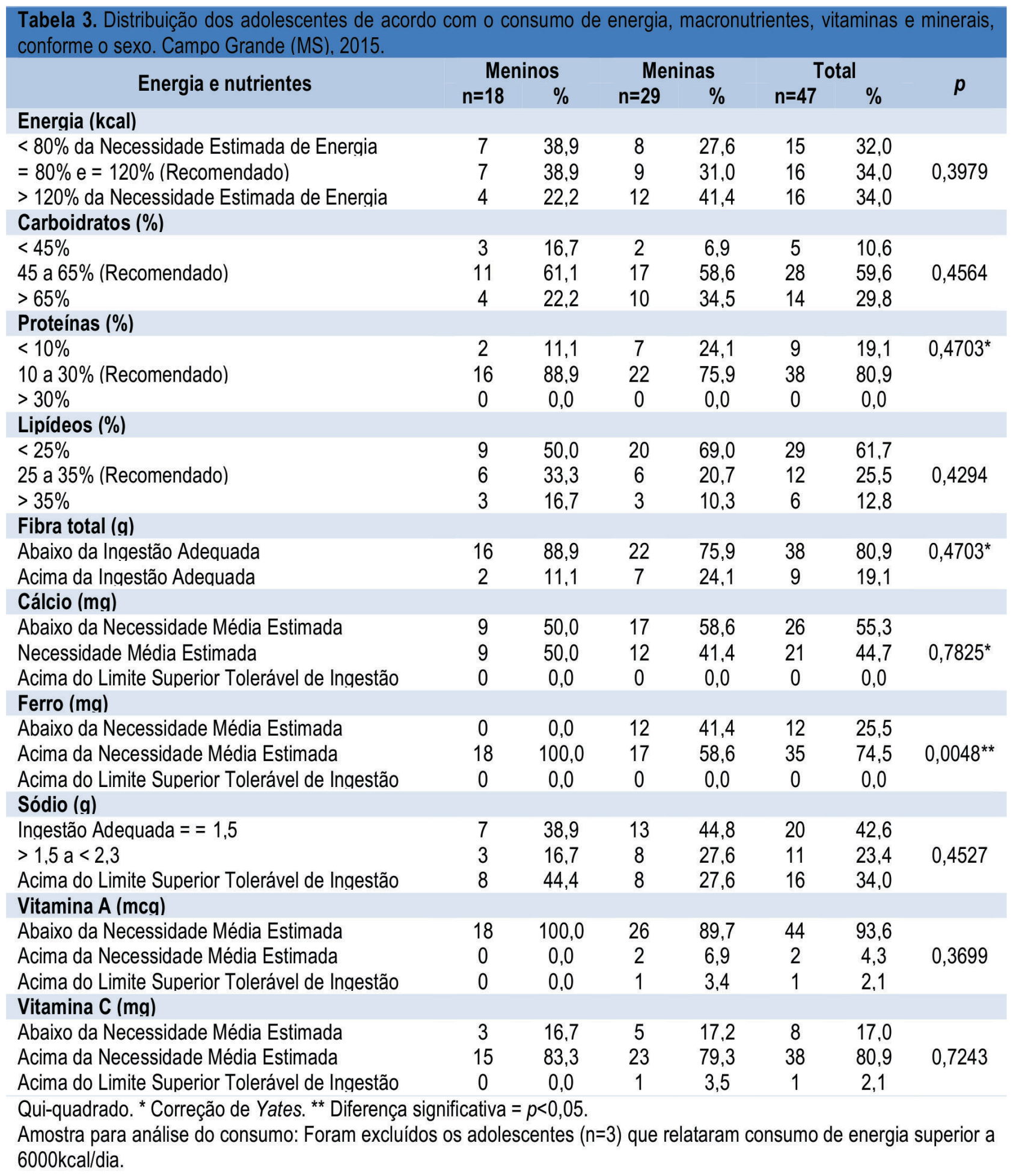




\begin{tabular}{|c|c|c|c|c|c|c|}
\hline \multirow{3}{*}{ Nutrientes } & \multicolumn{6}{|c|}{$\begin{array}{l}\text { Consumo de alimentos de acordo com o sexo e estado nutricional } \\
\text { (Média } \pm \text { DP) }\end{array}$} \\
\hline & \multicolumn{2}{|c|}{ Meninos } & \multicolumn{4}{|c|}{ Meninas } \\
\hline & $\begin{array}{l}\text { Eutrofia } \\
(n=13)\end{array}$ & $\begin{array}{l}\text { Excesso de peso } \\
\qquad(n=5)\end{array}$ & $p$ & $\begin{array}{c}\text { Eutrofia } \\
(n=20)\end{array}$ & $\begin{array}{l}\text { Excesso de peso } \\
\qquad(n=8)\end{array}$ & $p$ \\
\hline Eneraia (kcal) & $2831.2 \pm 1249.7$ & $2562.7 \pm 739.6$ & 0.7301 & $2613.5 \pm 1016.0$ & $2266.5 \pm 588.6$ & 0.4924 \\
\hline $\begin{array}{l}\text { Carboidratos } \\
(\%)\end{array}$ & $58,6 \pm 9,2$ & $56,5 \pm 19,6$ & 0,5877 & $59,2 \pm 10,2$ & $68,3 \pm 9,9$ & $0,0419^{*}$ \\
\hline Proteínas (\%) & $16,8 \pm 5,0$ & $17,5 \pm 8,6$ & 0,8825 & $15,7 \pm 5,2$ & $13,1 \pm 5,3$ & 0,2972 \\
\hline Lipídeos (\%) & $24,6 \pm 8,8$ & $25,9 \pm 12,4$ & 0.5217 & $25,1 \pm 7,5$ & $18,6 \pm 6,2$ & $0,0473^{*}$ \\
\hline Fibra total (q) & $30.1 \pm 14.1$ & $18.3 \pm 5.4$ & $0,0433^{*}$ & $25.1 \pm 18.8$ & $20,0 \pm 7,1$ & 0.9190 \\
\hline Cálcio (ma) & $1084,0 \pm 562,7$ & $1212,7 \pm 833,6$ & 0,8053 & $921,7 \pm 535.8$ & $1034,0 \pm 469.2$ & 0,5417 \\
\hline Ferro (ma) & $13.5 \pm 6.2$ & $12,5 \pm 3.8$ & 0.8053 & $12,0 \pm 6.9$ & $8.3 \pm 3.9$ & 0.1092 \\
\hline Sódio (q) & $2219.6 \pm 1217,7$ & $2651.9 \pm 1119.9$ & 0.4597 & $1937,0 \pm 1122,9$ & $2046.1 \pm 1514.5$ & 0.7219 \\
\hline $\begin{array}{l}\text { Vitamina A } \\
\text { (mca) }\end{array}$ & $244,4 \pm 124,3$ & $340,2 \pm 129,7$ & 0,1265 & $751,5 \pm 2442,8$ & $200,1 \pm 215,3$ & 0,5759 \\
\hline $\begin{array}{l}\text { Vitamina C } \\
\text { (ma) }\end{array}$ & $424,9 \pm 362,0$ & $459,4 \pm 566,6$ & 0,8825 & $573,3 \pm 696,0$ & $636,6 \pm 386,8$ & 0,2855 \\
\hline
\end{tabular}

* Teste de Mann-Whitnev ( $0<0,05)$.

\begin{tabular}{|c|c|c|c|c|c|c|c|c|}
\hline \multicolumn{9}{|c|}{$\begin{array}{l}\text { Tabela 5. Hábitos alimentares dos adolescentes ( } \mathrm{n}=50 \text { ) de acordo com a frequência de consumo semanal, Campo Grande } \\
\text { (MS), } 2015 \text {. }\end{array}$} \\
\hline 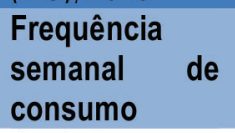 & $\begin{array}{c}\text { Feijão } \\
(\%)\end{array}$ & $\begin{array}{c}\text { Salada } \\
\text { Crua* } \\
(\%)\end{array}$ & $\begin{array}{c}\text { Frutas } \\
(\%)\end{array}$ & $\begin{array}{c}\text { Carne } \\
\text { Vermelha } \\
(\%)\end{array}$ & $\begin{array}{l}\text { Carne de } \\
\text { Frango } \\
(\%)\end{array}$ & $\begin{array}{l}\text { Refrigerante ou } \\
\text { Suco Artificial } \\
(\%)\end{array}$ & $\begin{array}{l}\text { Leite } \\
(\%)\end{array}$ & $\begin{array}{c}\text { Doces } \\
(\%)\end{array}$ \\
\hline 1 a 2 dias & 10,0 & 24,0 & 32,0 & 12,0 & 46,0 & 16,0 & 24,0 & 20,0 \\
\hline 3 a 4 dias & 18,0 & 18,0 & 28,0 & 22,0 & 38,0 & 36,0 & 22,0 & 20,0 \\
\hline 5 a 6 dias & 14,0 & 20,0 & 16,0 & 24,0 & 8,0 & 28,0 & 14,0 & 22,0 \\
\hline Todos os dias & 44,0 & 18,0 & 12,0 & 38,0 & 0,0 & 12,0 & 26,0 & 30,0 \\
\hline Quase nunca & 10,0 & 14,0 & 12,0 & 0,0 & 8,0 & 4,0 & 8,0 & 6,0 \\
\hline Nunca & 4.0 & 6.0 & 0.0 & 4,0 & 0.0 & 4.0 & 6.0 & 2,0 \\
\hline
\end{tabular}

* Salada de Alface e Tomate ou Verdura crua.

de carboidratos. Os autores argumentam que a diminuição da quantidade de um nutriente específico na dieta, altera a distribuição de outros componentes. Essa alteração na distribuição entre carboidratos e lipídeos, caso persista rotineiramente, com consumo excessivo, especialmente de carboidratos na forma de açúcares simples, poderá refletir em ganho de gordura corporal $^{3}$. Na presente pesquisa, o consumo de produtos fonte de carboidratos pode ser observado a partir das questões referentes aos hábitos alimentares, tendo em vista proporção importante de adolescentes que manifestaram frequência semanal elevada de consumo de doces e refrigerantes $(40 \%$ e $52 \%$ dos adolescentes informaram consumo de refrigerantes e doces de 5 a 7 vezes/semana, respectivamente).

Quanto à ingestão de carboidratos, complementando a questão de distribuição entre carboidratos e lipídeos, segundo estudo com adolescentes é comum que carboidratos sejam consumidos em excesso, visto o alto nível de açúcar na dieta, provindo das bebidas açucaradas que substituem outras opções mais saudáveis como o leite, caracterizando um padrão alimentar inadequado e de risco à saúde ${ }^{3}$. Em 2015 a Organização Mundial da Saúde reduziu a recomendação para ingestão de açúcares de $10 \%$ para $5 \%$ das calorias ingeridas, fundamentando-se no fato de que o consumo excessivo tem se mostrado fortemente associado ao desenvolvimento de obesidade e de $\mathrm{DCNT}^{22}$, o que potencializa a importância da orientação nutricional entre os jovens.

Ao considerar a ingestão de energia de acordo com o estado nutricional e sexo não foi constatada diferença significativa, contudo algumas considerações chamam a atenção. Ao comparar a ingestão média de energia com o valor médio da Necessidade Estimada de Energia (calculada de acordo com a amostra deste 
estudo) é possível constatar que: o valor médio de energia ingerido pelos meninos eutróficos representa 98,1\% em relação à Necessidade Estima de Energia, enquanto que entre as meninas eutróficas, este valor alcança 128,6\%; entre os meninos com excesso de peso a ingestão média representa $72,4 \%$ da necessidade e, entre as meninas, $80,8 \%$. Portanto, neste estudo os adolescentes, de ambos os sexos, com excesso de peso, relataram consumo de energia inferior ao valor médio recomendado. Uma possível explicação pode decorrer do constrangimento dos adolescentes em descrever sua dieta detalhadamente, sabendo que continha falhas e/ ou excessos ou porque não estavam plenamente conscientes da quantidade de alimentos ingerida ${ }^{3}$. O mesmo foi observado em um estudo conduzido com adolescentes, com idades entre 14 e 19 anos, matriculados em escolas estaduais de Ribeirão Preto ${ }^{23}$. Estudo realizado com adolescentes com idade média de 16 anos constatou que adolescentes obesos tem 5 vezes mais chances de subnotificar a ingestão energética do que adolescentes com peso normal ${ }^{24}$.

O cálcio, relevante durante a adolescência por contribuir para a mineralização óssea ${ }^{25}$, destacou-se em decorrência do consumo insuficiente por parcela substancial de adolescentes (55\%) desta pesquisa. Estudo com adolescentes de 10 a 19 anos revelou consumo insuficiente por $56,75 \%$ dos participantes ${ }^{2}$. Neste sentido, considera-se que o consumo de outros alimentos, em substituição ao leite, represente um fator cada vez mais presente entre os jovens, sendo que o consumo de refrigerantes 3 vezes ou mais na semana mostrou-se associado com menor adequação da ingestão de cálcio em adolescentes ${ }^{26}$.

$\mathrm{O}$ consumo insuficiente de vitamina A destacou-se para ambos os sexos, com proporções importantes (100\% e 89,7\%, para os meninos e meninas, respectivamente). Esse nutriente desempenha funções essenciais para a saúde, atuando no crescimento, na visão, integridade dos tecidos, nos dentes e no processo de reprodução. Uma vez envolvida nesses processos, a vitamina A se torna indispensável na adolescência ${ }^{27}$.

Estudo realizado entre crianças vivendo na Etiópia, com idades entre 4 e 15 anos, encontrou 29,3\% de deficiência de vitamina A sérica. Após coleta de dados dietéticos verificou-se associação significativa entre $o$ baixo consumo de alimentos fonte desse nutriente e a deficiência nos níveis séricos ${ }^{28}$.

No presente estudo, alguns alimentos característicos da alimentação sul-mato-grossense, fontes de vitamina $\mathrm{A}$, poderiam contribuir para potencializar a ingestão, entre eles o pequi, a manga, a bocaiuva e o milho verde, os quais poderiam compor a alimentação com maior frequência. Para isso, estratégias educativas de estímulo ao consumo poderiam ser implementadas em diversos cenários, como em Unidades Básicas de Saúde, Núcleos de Apoio à Saúde da Família, Centros de Educação Infantil e Escolas Públicas, com o intuito de integrar a família no processo educativo.

O consumo de ferro mostrou perfil favorável, pois a quantidade média de ferro ingerida foi superior à recomendada, independente do estado nutricional e sexo. Porém, enquanto $100 \%$ dos meninos informaram consumo acima da recomendação, cerca de $58 \%$ das meninas apresentaram tal categoria de consumo. A partir das questões referentes aos hábitos alimentares constatou-se que os adolescentes consomem alimentos fonte de ferro com maior frequência que as meninas, como o feijão e carnes em geral (principalmente vermelha). Notou-se também elevado consumo de alimentos industrializados, elaborados com farinhas de trigo e milho fortificadas com ferro e outros minerais. Desse modo, o consumo rotineiro desses alimentos pode aumentar a ingestão de ferro.

A ingestão do mineral ferro foi investigada em crianças e adolescentes de 6 a 18 anos por meio da utilização de diário alimentar de $3 \operatorname{dias}^{29}$. A prevalência de inadequação entre os meninos de 14 a 18 anos foi inferior $(6,9 \%)$ em relação às meninas $(25,3 \%)$. Em outro estudo, 58,5\% dos meninos apresentaram consumo conforme a recomendação, enquanto que $37,3 \%$ das meninas alcançaram o recomendado. ${ }^{1}$ No presente estudo, embora com consumo médio favorável, 41,4\% das meninas apresentaram consumo insuficiente em relação à Necessidade Média Estimada. Apesar dos estudos mencionados analisarem populações da mesma faixa etária, mostram realidades culturais distintas.

A análise do consumo de fibras mostrou que $80,9 \%$ dos adolescentes da presente pesquisa apresentou consumo abaixo da recomendação. Complementando este fato, constatou-se que $12 \%$ e $18 \%$ dos adolescentes afirmaram consumir frutas e saladas cruas diariamente, o que pode auxiliar a compreensão do reduzido consumo de fibras. Adolescentes tendem a ingerir menos frutas e legumes, dando preferência para alimentos com maior quantidade de carboidratos refinados e gorduras ${ }^{3}$, o que também pode ser notado neste estudo, em que $30 \%$ dos adolescentes mencionaram consumo diário de doces.

Outro dado preocupante é o elevado consumo de sódio entre os adolescentes. Nesta faixa de idade, a ingestão excessiva de alimentos industrializados, ricos também em sódio, é muito comum ${ }^{30}$. A ingestão adequada desse mineral auxilia na redução da pressão sanguínea, diminui o risco de doenças cardiovasculares, acidente vascular e ataque cardíaco $^{31}$. Desse modo, 
considerando os valores médios registrados de acordo com o estado nutricional, os adolescentes deste estudo deveriam reduzir, em média, aproximadamente $700 \mathrm{mg}$ por dia para alcançarem a quantidade adequada, ou seja, redução de cerca de $30 \%$ em relação ao ingerido para manter o consumo em $1500 \mathrm{mg}$.

A investigação dos hábitos alimentares considerados marcadores de uma alimentação adequada/saudável, bem como dos marcadores desfavoráveis, apresentou resultados relacionados a um perfil de consumo que, quando adotados rotineiramente, poderá colocar os adolescentes em risco nutricional. Apenas 44\% e 26\% dos adolescentes, respectivamente, manifestaram consumir feijão e leite diariamente, o que ressalta um possível risco de déficit de nutrientes específicos, como ferro e cálcio. Dados da Pesquisa Nacional de Saúde do Escolar $^{32}$ revelaram que 69,9\% dos escolares consumiam feijão 5 dias ou mais na semana. Enquanto que o leite era consumido por 51,5\% dos adolescentes nesta mesma frequência. Vale ressaltar que o Guia Alimentar para População Brasileira ${ }^{33}$ recomenda a ingestão diária de feijão visto que a associação desse alimento com o arroz constitui uma escolha nutricionalmente balanceada de aminoácidos essenciais. Sobre o leite, o consumo dos sul-matogrossenses mostrou-se ainda mais desfavorável, por integrar diariamente a alimentação de reduzida parcela de adolescentes.

Com relação à ingestão de frutas, legumes e verduras, $8 \%$ e $12 \%$ dos adolescentes referiram consumir saladas e frutas todos os dias. O baixo consumo desses grupos alimentares pode ser reflexo, em parte, da influência da mídia sobre os jovens. Estudo feito com adolescentes constatou que 34\% deles destinavam mais de 4 horas de tempo de televisão e $36 \%$ mais de 4 horas de tempo de computador por dia. ${ }^{34}$ Por meio das propagandas, notadamente as veiculadas pela televisão, são difundidas mensagens em relação aos alimentos/ produtos industrializados atribuindo-lhes qualidades superiores, e por vezes equivocadas, quanto ao conteúdo nutricional que realmente apresentam. Além disso, propagandas comumente valorizam produtos industrializados e raramente abordam a importância de alimentos in natura, ainda mais por meio de estratégias diferenciadas que despertem o interesse do adolescente. Outra situação a ser considerada é o próprio hábito alimentar da família, influenciado pelo tempo para o preparo, facilidade de aquisição, durabilidade dos alimentos in natura e renda.

Outro fator que influencia o consumo de alimentos é o preço. No ano de 2015, o valor da cesta básica em Campo Grande (MS) aumentou 22,78\%, um dos maiores aumentos do país ${ }^{35}$. O maior preço da cesta básica possivelmente pode associar-se à redução do consumo de alimentos in natura. Com a aquisição de alimentos comprometida, faz-se necessário que o responsável pela alimentação da família tenha acesso a conhecimentos sobre a importância do consumo adequado de frutas e verduras, a fim de contemplar minimamente a disponibilidade de nutrientes importantes para a prevenção de doenças em todas as faixas de idade. Suplementos ou medicamentos com nutrientes isolados não tem a mesma garantia de proteção ${ }^{33}$. A ingestão de 5 porções de frutas e vegetais por dia (400 g) reduz o risco de DCNT e garante adequada ingestão diária de fibra dietética ${ }^{36}$.

Entre escolares brasileiros, oriundos de amostra representativa de 26 capitais e do Distrito Federal, participantes da Pesquisa Nacional de Saúde do Escolar (2013), foi observado que o consumo de doces em 5 dias ou mais na semana foi de $41,3 \%$. O consumo de refrigerantes também se mostrou elevado $(33,2 \%)^{32}$. Na presente pesquisa, 30\% dos adolescentes referiram consumo diário de doces e $36 \%$ mencionaram consumir refrigerantes diariamente. É reconhecido que o elevado consumo de alimentos ricos em açúcares poderá repercutir na redução de consumo de alimentos mais saudáveis, agregando assim maior risco para o desenvolvimento de desequilíbrios metabólicos e excesso de gordura corporal em idade precoce ${ }^{22}$.

Em relação ao registro do consumo de alimentos pelos adolescentes, o autopreenchimento foi considerado fator limitante neste estudo, pois foram observadas dificuldades inerentes ao pouco conhecimento dos adolescentes sobre quantidade, tamanho de porções e nome de preparações. Todos os registros foram conferidos imediatamente após a aplicação, desse modo os erros foram corrigidos/ minimizados. Outra questão relevante diz respeito às desistências $(29,6 \%)$ ao longo do estudo, justificadas pelo remanejamento de alunos por troca de turno e cancelamento de matrículas. Alguns alunos sentiramse desconfortáveis no momento de aferir o peso, mesmo que em ambiente reservado, o que causou desistência nessa etapa.

\section{CONCLUSÃO}

A proporção de adolescentes com excesso de peso foi elevada neste estudo. As escolhas alimentares constatadas indicam consumo insuficiente de nutrientes essenciais (cálcio, vitamina A e fibras) e consumo expressivo de alimentos associados ao acúmulo de gordura corporal. Tendo em vista tratar-se de indivíduos jovens e em fase de intenso desenvolvimento, ressalta- 
se a relevância dos cuidados com os hábitos alimentares e atividades físicas. Assim, acredita-se que estudos com início no período final da infância, antes dos 10 anos de idade, além de proporcionarem maior participação/ adesão, possam representar possibilidades de acompanhamento específico, contínuo e permeados por estratégias de Educação Alimentar e Nutricional, fato que poderá permitir a detecção de associações de hábitos alimentares e de vida com o estado nutricional e, consequentemente, ampliar as possibilidades de ações efetivas de intervenção.

\section{REFERÊNCIAS}

1. Córdoba-Caro LG, Pérez LML, Preciado VG. Adecuación nutricional de la ingesta de los estudiantes de secundaria de Badajoz. Nutr Hosp. 2012; 27(4):1065-1071.

2. Deka MK, Malhotra AK, Yadav R, Gupta S. Dietary pattern and nutritional deficiencies among urban adolescents. J Family Med Prim Care. 2015; 4(3):364368.

3. Pinho L, Botelho ACC, Caldeira AP. Associated factors of overweight in adolescents from public schools in northern Minas Gerais state, Brazil. Rev Paul Pediatr. 2014; 32(2):237- 243.

4. American Academy of Pediatrics. Committee on Nutrition. Prevention of pediatric overweight and obesity. 2003; 112(2):424-430.

5. Silva DAS, Silva RJS. Associação entre prática de atividade física com consumo de frutas, verduras e legumes em adolescentes do Nordeste do Brasil. Rev Paul Pediatr. 2015; 33(2):167-173.

6. World Health Organization [homepage]. Physical activity [acesso em: 22 fev 2016]. Disponível em: http:// www.who.int/topics/physical_activity/en/

7. ABEP (Associação Brasileira de Empresas de Pesquisa). Critério de Classificação Econômica Brasil, 2014 [acesso em: 22 jan 2016]. Disponível em: http:// www.abep.org/criterio-brasil

8. World Health Organization [homepage]. Growth reference data for 5-19 years. WHO Reference 2007 [acesso em: 24 fev 2016]. Disponível em: http:// www.who.int/growthref/en/

9. Farias Júnior JC, Lopes AS, Mota J, Hallal PC. Prática de atividade física e fatores associados em adolescentes do Nordeste do Brasil. Rev Saúde Públ. 2012; 46(3):505-515.

10. Biddle S, Sallis J, Cavill N. Young and active? Young people and health-enhancing physical activity evidence and implications. Health Education Authority, London; 1998.

11. Fisberg RM, Martini LA, Slater B. Métodos de inquéritos alimentares. In: Fisberg RM, Slater B, Marchioni DML, Martini LA, editores. Inquéritos alimentares: métodos e bases científicos. Barueri, SP: Manole; 2005. p. 1-31.

12. Institute of Medicine. Dietary Reference Intakes for Vitamin A, Vitamin K, Arsenic, Boron, Chromium, Copper, lodine, Iron, Manganese, Molybdenum, Nickel, Silicon, Vanadium, and Zinc. Washinghton, DC: National Academy Press; 2000.

13. Institute of Medicine. Dietary Reference Intakes for Vitamin C, Vitamin E, Selenium, and Carotenoids. Washinghton, DC: National Academy Press; 2001.
14. Institute of Medicine. Dietary Reference Intakes for Water, Potassium, Sodium, Chloride, and Sulfate. Washinghton, DC: National Academy Press; 2004.

15. Institute of Medicine. Dietary Reference Intakes for Energy, Carbohydrate, Fiber, Fat, Fatty Acids, Cholesterol, Protein, and Amino Acids (Macronutrients). Washinghton, DC: National Academy Press; 2005.

16. Institute of Medicine. Dietary Reference Intakes for Calcium and Vitamin D. Washington, D.C.: National Academy Press, nov. 2011.

17. Institute of Medicine. Sodium intake in populations.Assessment of evidence. Washinghton, DC: National Academy Press; 2013.

18. Brasil. Ministério da Saúde. Secretaria de Vigilância em Saúde. Departamento de Vigilância de Doenças e Agravos não Transmissíveis e Promoção de Saúde. Vigitel Brasil 2012: vigilância de fatores de risco e proteção para doenças crônicas por inquérito telefônico. Brasília: Ministério da Saúde, 2013.

19. Brasil. Ministério da Saúde. Ministério do Planejamento, Orçamento e Gestão. Instituto Brasileiro de Geografia e Estatística. Pesquisa de Orçamentos Familiares 20082009. Antropometria e Estado Nutricional de Crianças, Adolescentes e Adultos no Brasil. Rio de Janeiro; 2010.

20. Lima MCC, Romaldini CC, Romaldini JH. Frequency of obesity and related risk factors among school children and adolescents in a low-income community. A crosssectional study. São Paulo Med J. 2015; 133(2):125130.

21. World Health Organization [homepage]. Obesity [acesso em: 24 fev 2016]. Disponível em: http://www.who.int/ topics/obesity/en/

22. World Health Organization. Information note about intake of sugars recommended in the WHO guideline for adults and children [acesso em: 24 fev 2016]. Disponível em: http://www.who.int/nutrition/ p u b I i c a t i ons/guideli nes/ sugar_intake_information_note_en.pdf?ua $=1$

23. Vieira MV, Del Ciampo IRL, Del Ciampo LA. Hábitos e consumo alimentar entre adolescentes eutróficos e com excesso de peso. Rev Bras Crescimento Desenvolv Hum. 2014; 24(2):157-162.

24. Santos LC, Pascoal MN, Fisberg M, Cintra IP, Martini LA. Notificação imprecisa da ingestão energética na dieta de adolescentes. J Pediatr. 2010; 86(5):400-404.

25. Golden NH, Abrams SA, Committee on Nutrition. Optimizing bone health in children and adolescents. Pediatrics. 2014; 134(4):1229-1243. 
26. Oliveira CF, Silveira CR, Beghetto M, Mello PD, Mello ED. Assessment of calcium intake by adolescents. Rev Paul Pediatr. 2014; 32(2):216-220.

27. Moreira AVB, Sant'ana HMP. Vitaminas. In: Silva SMCS, Mura JDP, editores. Tratado de alimentação, nutrição e dietoterapia. São Paulo: Roca; 2014. p. 82-84.

28. Herrador Z, Sordo L, Gadisa E, Buño A, Gómez-Rioja R, Iturzaeta JM, de Armas LF, Benito A, Aseffa A, Moreno J, Cañavate C, Custodio E. Micronutrient deficiencies and related factors in school-aged children in Ethiopia: a cross-sectional study in Libo Kemkem and Fogera districts, Amhara Regional State. PLoS One. 2014; 9(12):1-20

29. Tornaritis MJ, Philippou E, Hadjigeorgiou C, Kourides YA, Panayi A, Savva SC. A study of the dietary intake of Cypriot children and adolescents aged 6-18 years and the association of mother's educational status and children's weight status on adherence to nutritional recommendations. BMC Public Health. 2014; 14(13):111.

30. Barreto Neto AC, Andrade MIS, Lima VLM, Diniz AS. Peso corporal e escores de consumo alimentar em adolescentes no nordeste brasileiro. Rev Paul Pediatr. 2015; 33(3):318-325.

31. World Health Organization [homepage]. Salt reduction [acesso em: 24 fev 2016]. Disponível em: http:// www.who.int/mediacentre/factsheets/fs393/en/
32. Brasil. Ministério do Planejamento, Orçamento e Gestão, Instituto Brasileiro de Geografia e Estatística - IBGE. Pesquisa Nacional de Saúde do Escolar. Rio de Janeiro, 2013.

33. Brasil. Ministério da Saúde. Guia alimentar da população brasileira. Brasília, 2005.

34. Farias ES, Santos AP, Farias Júnior JC, Ferreira CRT, Carvalho WRG, Gonçalves EM, Guerra Júnior G. Excesso de peso e fatores associados em adolescentes. Rev Nutr. 2012; 25(2):229-236.

35. Brasil. Departamento Intersindical de Estatísticas e Estudos Socioeconômicos - DIEESE. Valor da cesta básica aumenta em todas as capitais em 2015. São Paulo, 2016.

36. World Health Organization [homepage]. Healthy diet [acesso em: 24 fev 2016]. Disponível em: http:// www.who.int/mediacentre/factsheets/fs394/en/

\section{Correspondência}

Giovana Eliza Pegolo

Universidade Federal de Mato Grosso do Sul (UFMS)

Cidade Universitária, $\mathrm{S} / \mathrm{N}$, Universitário.

Campo Grande - Mato Grosso do Sul - Brasil

CEP: $79070-900$

E-mail: giovana.pegolo@hotmail.com

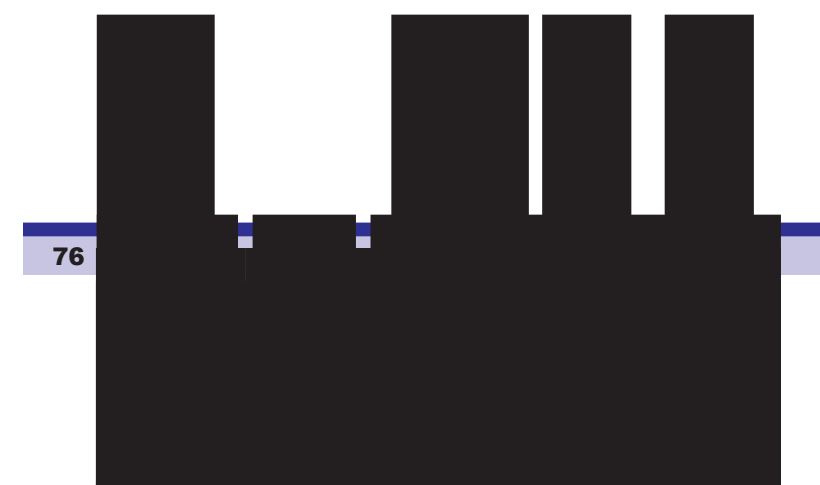

